\title{
Glycobiologie et cellules sanguines
}

La glycobiologie, c'est-à-dire l'étude des glucides et des glycoconjugués, connaît un vif renouveau depuis qu'il a été démontré que des glycanes constituaient la cible de protéines d'adhérence intervenant, notamment, dans la domiciliation (homing) des lymphocytes et dans nombre de fonctions leucocytaires. Les sélectines constituent l'une de ces classes de protéines d'adhérence qui incluent aussi la protéine H-CAM (ou CD44) et d'autres molécules moins bien caractérisées. Deux affections hématologiques congénitales, le syndrome de Wiskott-Aldrich et l'anémie congénitale dysérythropoiétique de type 2 , sont associées à des anomalies très spécifiques d'enzymes intervenant dans la synthèse ou la dégradation des glycanes, des transférases dans le premier cas et une $\alpha$ mannosidase dans le second.

\section{Dominique Néel Michèle Aubery Christian Derappe}

\section{ADRESSE}

D. Néel : maître de conférences des universités, praticien hospitalier. M. Aubery : directeur de recherche à l'Inserm. C. Derappe : chargé de recherche à l'Inserm. Inserm U. 180, faculté de médecine, 45, rue des Saints-Pères, 75006 Paris, France.

\section{TIRÉS A PART}

D. Néel. es glycanes sont omniprésents à la surface des cellules eucaryotes. Ils sont soit liés aux protéines pour former les glycoprotéines, soit liés à certains lipides pour former les glycolipides. La fixation des structures glucidiques aux protéines peut se faire de deux façons : la liaison $\mathrm{N}$ glycosidique, qui résulte de la fixation d'une chaîne saccharidique à un résidu d'asparagine inclus dans une séquence signal asparagine-Xthréonine ou sérine, et la liaison $\mathrm{O}$ glycosidique, formée par la fixation de glycanes sur un résidu de thréonine ou de sérine. La $\mathrm{N}$-glycosylation commence dans le réticulum endoplasmique au cours de la traduction pour se poursuivre dans les différents compartiments du Golgi, alors que la O-glycosylation est uniquement post- traductionnelle et se déroule dans le Golgi (figure 1, p. 234) [1].

Ces structures glycaniques sont d'une extrême diversité et nécessitent pour leur synthèse un équipement enzymatique très complexe. De plus, on remarquera que certaines séquences osidiques sont spécifiques de la nature de la molécule vectrice ou du type de liaison. Cependant, leur rôle biologique reste encore pour une bonne part mal connu. Certes les remarquables travaux de l'équipe de S. Kornfeld avaient démontré le rôle d'oligosaccharides $\mathrm{N}$-liés contenant du mannose-6-phosphate dans le ciblage des enzymes lysosomiales, de leur lieu de synthèse dans le réticulum jusqu'à l'organite de destination [2], mais cela ne concernait pas la grande masse des glycoprotéines et glycolipides membranaires. Les rôles dévolus 


\section{RÉFÉRENCES}

1. Kornfeld R, Kornf eld S. Assembly of Nlinked oligosaccharides. Annu Rev Biochem 1985 ; 54 : 631-4.

2. Dahms NM, Lobel P, Kornfeld S. Mannose-6-phosphate receptors and lysosomal enzyme targeting. J Biol Chem 1989 ; 264: 12115-8.

3. Yednock TA, Rosen ST. Lymphocyte homing. Adv in Immunol $1989 ; 44$ : 313-78. 4. Springer TA. Adhesion receptor of the immune system Nature 1990 ; 346 : 425-34. 5. Lasky LA. Lectin cell adhesion molecules (LEC-CAMs); a new family of cell adhesion proteins involved with inflammation. J Cell Biol 1991 ; 45 : 139-46.

6. Berg EL, Robinson MK, Warnocks RA, Butcher EC. The human peripheral lymph node vascular addressin is a ligand for LECAM1, the peripheral lymph node homing receptor. J Cell Biol 1991; 114 : 343-9.

7. Philipps ML, Nudelman E, Gacta FCA, et al. ELAM1 mediates cell adhesion by recognition of a carbohydratc ligand, sialylLc $^{\mathbf{x}}$. Science $1990 ; 250: 1130-2$.

8. Lowe JB, Stoolman LM, Nair RP, Larsen RD, Berhend JL, Marks RM. ELAM1 dependant cell adhesion to vascular endothelium determined by a transfected human glycosyltransferase cDNA. Cell 1990; 63 : 1349-56.

9. Goelz SE, Hession C, Goff D, et al. ELFT : a gene that directs the expression of an ELAM1 ligand. Cell 1990; 63: 1349-56.

10. Timeyer M, Swicdler SJ, Ishira M, et al. Carbohydrate ligands for endothelial leucocytes adhesion molecule 1. Proc Natl Acad Sci USA 1991; 88 : 1138-42.

11. Brandley BK, Swicdler SJ, Robbins PW. Carbohydrate ligands of the LEC cell adhesion molecules. Cell 1990 ; 63 : 861-3. 12. Culty M, Miyake K, Kincade PW, Silorski E, Butcher EC, Underhill C. The hyaluronate receptor is a member of the CD44 (H-CAM) family of cell surface glycoproteins. J Cell Biol 1990; 111 : 2765-74. 13. Diamond MS, Stauton DE, Marlin SD, Springer TA. Binding of the integrin Mac1 (CD11b/CD18) to the third immunoglobulin like domain of ICAM1 (CD54) and its regulation by glycosylation. Cell $1991 ; 65$ : 961-71.

14. Merlu B, Néel D, Goussault Y, Charron DJ. Glycosylation of the human leukocyte locus A molecules is dependent on ccll type. Eur J Biochem $1989 ; 181$ : 755-60. 15. Higgins EA, Siminovitch KA, Zhuang D, Brockhausen I, Dennis JW. Aberrant Olinked oligosaccharide biosynthesis in lymphocytes and platelets from patients with the Wiskott-Aldrich syndrom. J Biol Chem 1991 ; 266 : 6280-90.

16. Feugeas JP, Néel D, Goussault $Y$, Derappe C. Glycosylation of the human erythrocyte glucose transporter : a minimum structure is required for glucose transport activity. Biochim Biophys Acta 1991; 1066 : 59-62.

17. Fukuda M. HEMPAS disease : a genetic defect in glycosylation. Glycobiology 1990 ;

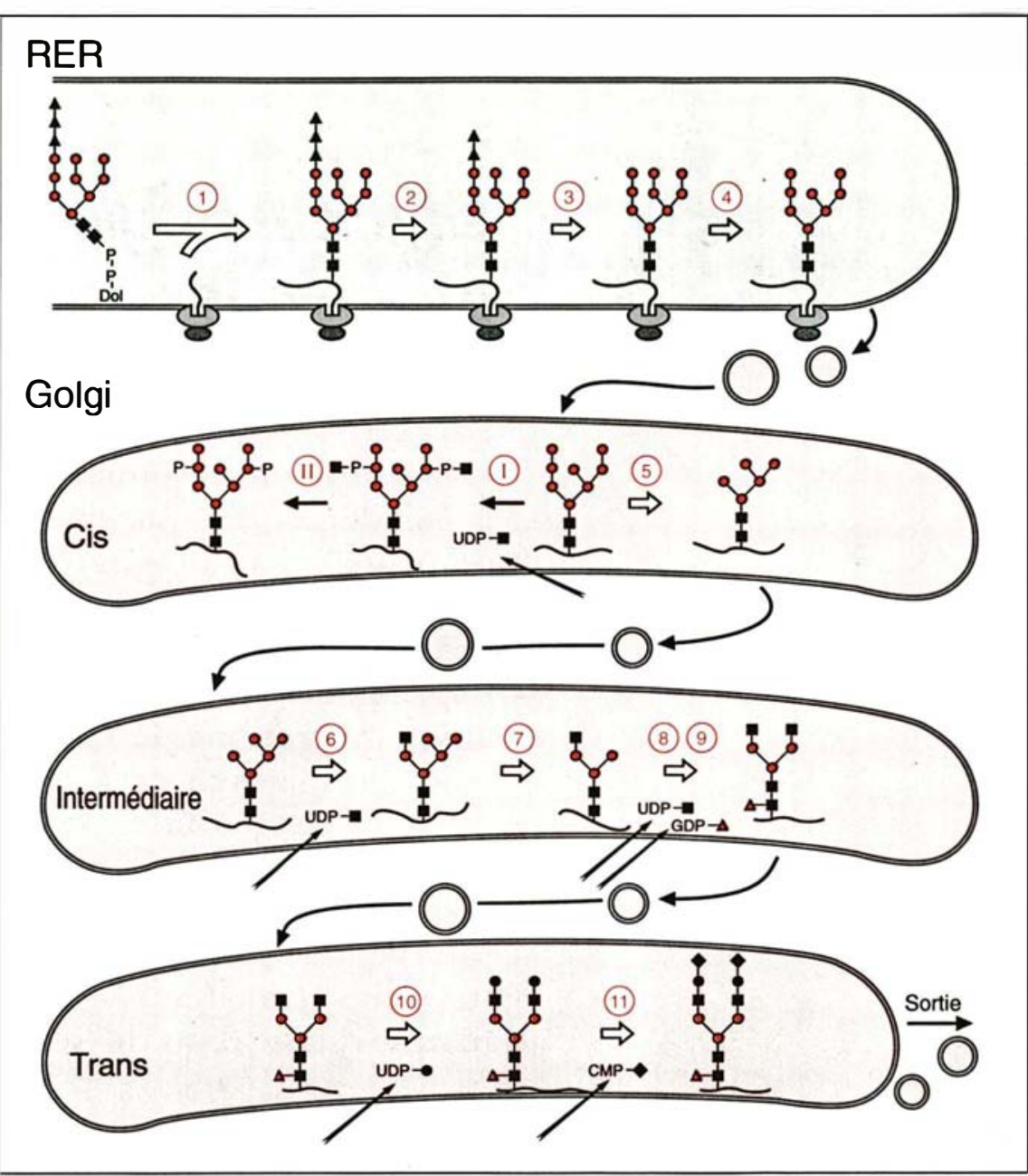

Figure 1. Représentation schématique du processus de maturation des oligosaccharides $\boldsymbol{N}$-liés (d'après [1]). Les réactions sont catalysées par les enzymes suivantes : (1) oligosaccharyltransférases ; (2) $\alpha$-glucosidase 1 ; (3) $\alpha$-glucosidase 11 ; (4) $\alpha$-1, 2-mannosidase du réticulum endoplasmique ; (I) $N$ acétylglucosaminylphosphotransférase ; (II) N-acétylglucosamine-1-phosphodiester $\alpha$-N-acétylglucosaminidase; (5) $\alpha$-mannosidase I du Golgi; (6) $\mathrm{N}$ acétylglucosaminyltransférase 1; (7) $\alpha$-mannosidase II du Golgi; (8) $\mathrm{N}$ acétylglucosaminyltransférase II; (9) fucosyltransférase; 110$)$ galactosyltransférase ; (11) sialyltransférase. Les symboles représentent les résidus suivants : $\mathbf{N}$-acétylglucosamine; mannose ; $\Delta$ glucose ; $\Delta$ fucose ; galactose ; acide sialique. RER : réticulum endoplasmique rugueux.

aux structures glucidiques des glycoprotéines semblaient se résumer à celui d'une protection contre l'action des protéases ou, dans certains cas, à celui du maintien d'une structure spatiale particulière, fonctions qui ne semblaient pas nécessiter la grande variété structurale observée.

C'est au cours de ces toutes dernières années que des travaux, concernant pour la plupart la biologie des cellules sanguines, ont suscité un regain d'intérêt pour la glycobiologie.
Ces recherches portaient sur un domaine jusque-là peu étudié, celui de l'adressage et de la recirculation des lymphocytes. Rappelons qu'un des maillons essentiels du système de défense immunitaire est l'ensemble des ganglions lymphatiques et autres structures lymphoïdes spécialisées disséminées au niveau de la peau, des tractus intestinal et urogénital, de façon à protéger l'organisme des antigènes pouvant y pénétrer par effraction, et qu'une des étapes clés de la 
réponse immune est la présentation de ces antigènes aux lymphocytes. Cclle-ci nécessite une circulation continuelle de ces cellules du sang aux organes lymphoïdes et de ceux-ci au sang, de façon que les antigènes rencontrent le plus rapidement possible les lymphocytes capables d'engendrer une réponse spécifique. Ce phénomène d'entrée ou de retour des lymphocytes de la circulation sanguine aux organes lymphoïdes, appelé homing, ou domiciliation, est souvent spécifique de structures lymphoïdes particulières ; ainsi certaines souspopulations de lymphocytes retourneront électivement vers les ganglions lymphatiques alors que d'autres se dirigeront uniquement vers les plaques de Peyer [3].

La voie d'entrée des lymphocytes du sang aux ganglions lymphatiques se fait grâce à une structure particulière de petits vaisseaux appelés high endothelial venules (HEV) où les cellules endothéliales sont de forme cubique au lieu d'avoir l'aspect aplati habituel. Les HEV ne sont présents qu'au niveau des organes lymphoïdes secondaires (ganglions lymphatiques, plaques de Peyer, amygdales, etc.), ccpendant on les trouve parfois au niveau d'un organe subissant une inflammation chronique caractérisée par l'infiltration de nombreux lymphocytes et polynucléaires. Une interaction entre les cellules des HEV et les lymphocytes apparaît nécessaire à l'cxtravasation de ces derniers. Cclle-ci a pu être visualisée par les cytologistes, qui ont montré que le premier contact entre les cellules se fait à partir de microvillosités lymphocytaires qui vont s'encastrer dans des dépressions de la cellule endothéliale, puis le lymphocyte s'cxtravase en passant entre deux cellules endothéliales et, finalement, se détache. Ainsi ce processus met en jeu successivement des stades d'adhérence et de "désadhérence ". En dehors des lymphocytes, d'autres cellules sanguines sont concernées par de tels phénomènes d'adhérence et/ou extravasation impliquant des cellules endothéliales, comme, par exemple, les polynucléaires ou même les plaquettes. Qucls sont les mécanismes moléculaires des liaisons endothélium-cellule sanguine? Plusieurs familles de molécules intervenant $\mathrm{m} / \mathrm{s} n^{\circ} 3$, vol. 8, mars 92

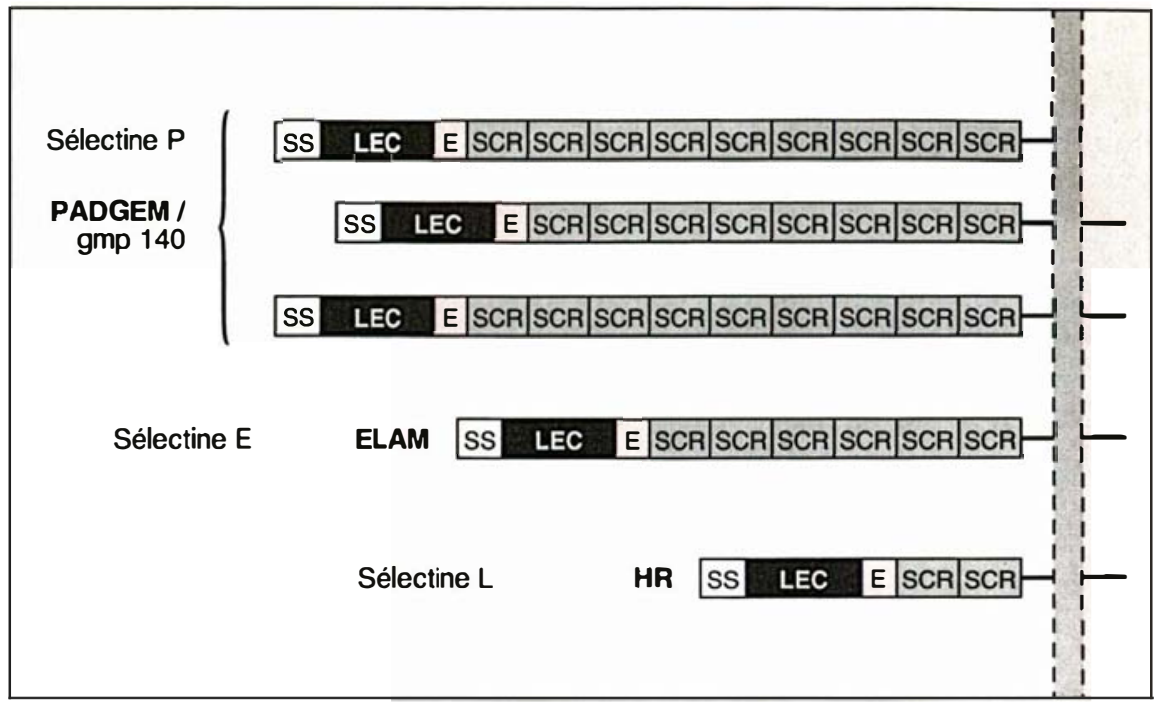

Figure 2. Structures des sélectines. Sont représentées : les structures du récepteur du homing (HR) ou sélectine $L$, de la sélectine $E$ et des formes complètes, délétées pour les séquences consensus ou solubles de la sélectine $P$ ou CD62. SS : séquence signal; LEC : lectine ; $E$ : séquence identique à l'EGF (epidermal growth factor) ; SCR : courte séquence consensus ; ELAM : endothelium lymphocyte adhesion molecule; GMP: granule membrane protein ; PADGEM : platelet activation dependent granule external membrane protein. (D'après [5].)

dans ces phénomènes d'adhérence, les adressines, ont été décrites [4] et parmi celles-ci un bon nombre d'entre elles reconnaissent comme ligands des structures oligosaccharidiques. Ce sont ces dernières qui nous intéresseront ici.

\section{Les sélectines}

La première famille est celle des sélectines, anciennement dénommées LEC-CAM, L pour lectrine, E pour EGF car elle ont une parenté structurale avec ce facteur de croissance, C pour complément car elles ont également une parenté structurale avec ce dernier (séquence consensus répétée en nombre variable selon les molécules) [5] et CAM pour cell adhesion molecule (figure 2). De plus, leur gène est situé, chez l'homme ainsi que chez la souris, sur le même chromosome, le $n^{\circ} 1$.

(1) La sélectine $\mathrm{L}$, récepteur de domiciliation des leucocytes, connue également sous le nom de LECCAM1, d'antigène Mel 14, mais aussi appelée G90 Mel, LAM1, Leu 8 ou Ly 22, a été d'abord isolée chez la souris. Il s'agit d'une glycoprotéine de $85-95 \mathrm{kDa}$ avec un point isoélcctrique acide de 4-4,5, exprimée sur l'ensemble des leucocytes. Elle inter- vient principalement dans la liaison des lymphocytes $\mathrm{T}$ aux HEV des ganglions lymphatiques, jouant donc un rôle important dans la recirculation de ces lymphocytes, mais aussi dans l'extravasation de l'ensemble des leucocytes qui se produit lors d'une réaction inflammatoire aiguë. En revanche, elle n'interviendrait pas dans la recirculation des lymphocytes au niveau des plaques de Peyer intestinales. La nature du déterminant glucidique reconnu par la sélectine $\mathrm{L}$ sur les HEV reste mal définie ; des trois sélectines décrites, c'est celle dont la nature du ligand glucidique est la moins bien connue, cependant la combinaison aux mannoses et aux fucoses de charges négatives provenant de sulfates, de phosphates ou, plus certainement, d'acide sialique en est un élément essentiel. La forme humaine de la sélectine L est reconnue par les anticorps monoclonaux Leu 8 et TQ1 et est hautement conservée par rapport aux formes murines. L'adhérence de la sélectine $L$ comme de l'ensemble des sélectines est dépendante du calcium. Un de ses ligands vient d'être caractérisé, il s'agit de l'adressine des ganglions lymphatiques périphériques (PNad),

(2) La sélectine $E$, auparavant appel'antigène MECA79 [6]. 
lée ELAM1 pour endothelium lymphocyte adhesion molecule, est une glycoprotéine de 110 kDa exprimée sur l'endothélium des $\mathrm{HEV}$ et des vaisseaux sanguins des zones tissulaires subissant une réaction inflammatoire. Diverses approches ont permis de caractériser les déterminants oligosaccharidiques reconnus par cette sélectine. Il a d'abord été démontré une corrélation entre l'adhérence de mutants de la lignée $\mathrm{CHO}$ sur des endothéliums activés et l'expression par ces mutants de la structure sialyl Lewis X figure 3) [7]. Une autre approche a été de transfecter des cellules $\mathrm{CHO}$ avec une 1-3/1-4 fucosyl transférase les rendant sialyl Lewis $\mathrm{X}$ positives et capables de lier la sélectine-E [8]. Enfin, il a été mis en évidence une nouvelle $\alpha$ 1-3 fucosyltransférase dont l'activité est suffisante pour conférer à ces variantes de la lignée $\mathrm{CHO}$, la capacité d'adhérence à la sélectine $\mathrm{E}$ [9]. La nature des structures moléculaires portant les déterminants sialyl Lewis $\mathrm{X}$ n'est pas encore clairement éluci-

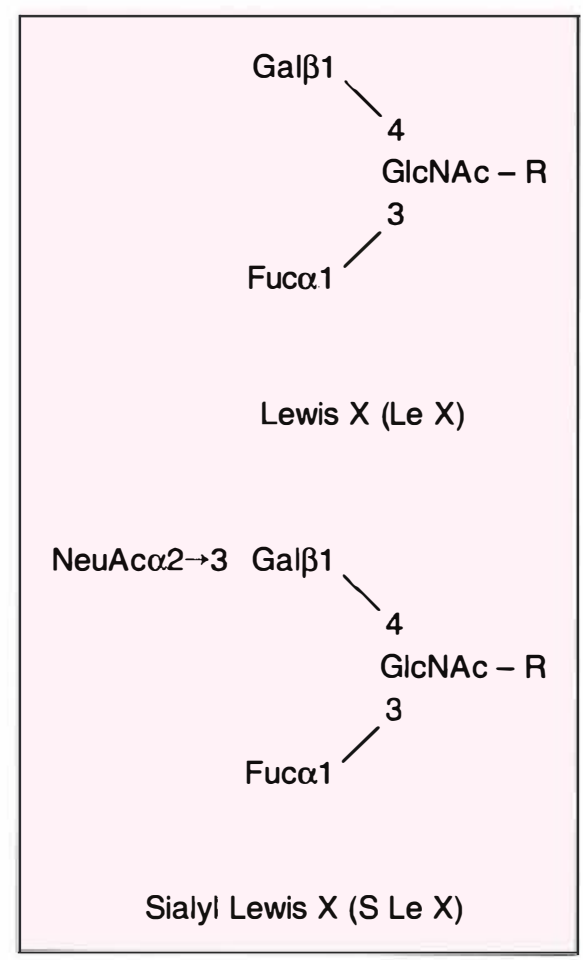

Figure 3. Structures oligosaccharidiques reconnues par la sélectine $E$ (sialyl-Lewis $X$ ) et la sélectine $P$ (Lewis $X$ ).

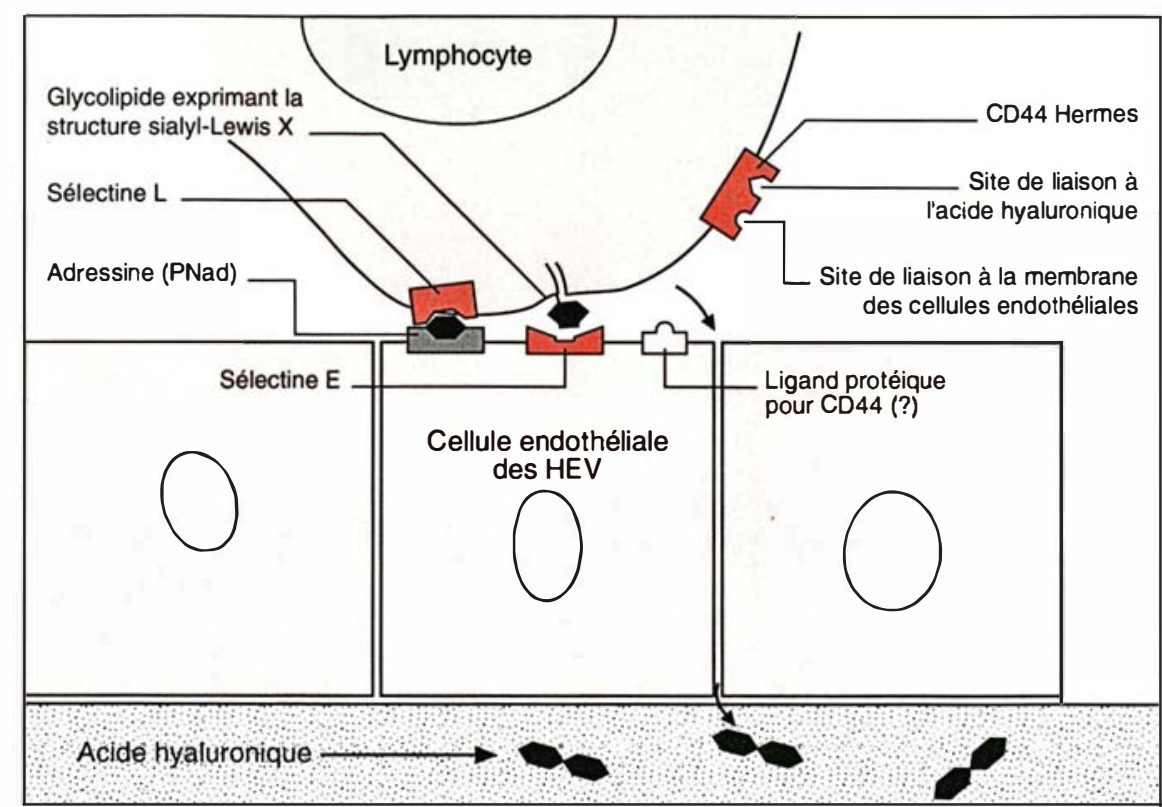

Figure 4. Représentation schématique des mécanismes pouvant impliquer des glycoconjugués au cours du homing des lymphocytes. Pour simplifier, les microvillosités présentes à la surface des lymphocytes et les dépressions de la cellule endothéliale n'ont pas été représentées. La flèche indique le chemin qui sera suivi par le lymphocyte. PNad: adressine des ganglions lymphatiques périphériques; HEV : high endothelial venules.

dée, mais il pourrait s'agir essentiellement de glycolipides [10].

(3) La sélectine $\mathbf{P}$ (CD62) [10], auparavant dénommée GMP140 pour granule membrane protein, présente dans les granules des plaquettes et les corps de Weibel Palade des cellules endothéliales, est la troisième sélectine actuellement connue. Rapidement mobilisable à la surface cellulaire lors de stimulations, elle interviendrait dans la liaison de certains leucocytes aux plaquettes ou aux cellules endothéliales. Son ligand oligosaccharidique serait apparenté au lactose-Nfucopentose (Lewis X).

Les déterminants glucidiques reconnus par ces trois sélectines présentent une parenté structurale : un fucose lié en $\alpha 1-3$ et, tout au moins pour les sélectines $L$ et $E$, la nécessaire présence d'un acide sialique [11].

\section{44 Hermès (H-CAM)}

Une autre molécule n'appartenant pas à la famille des sélectines mais reconnaissant également des déterminants de nature glucidique est impliquée dans les phénomènes de recirculation des lymphocytes; il s'agit de
CD44 (Hermès) appelé aussi $\mathrm{H}$ CAM. Cette glycoprotéine de 85 kDa est responsable de l'adhérence des cellules qui la porte à l'acide hyaluronique et au chondroïtine sulfate de la matrice extra-cellulaire. Présente à la surface des lymphocytes, elle jouerait un rôle dans l'adhérence des lymphocytes aux HEV des tissus muqueux lors du homing. Cependant, l'interaction entre CD44 et les HEV se ferait par un domaine de la molécule distinct de celui qui reconnaît l'acide hyaluronique, puisque l'excès d'acide hyaluronique ou le traitement par la hyaluronidase n'affectent pas la liaison lymphocyte-HEV et que certains anticorps anti-CD44 bloquent l'adhérence des lymphocytes aux HEV sans altérer l'interaction de ces mêmes cellules avec l'acide hyaluronique. En fait, CD44 pourrait avoir deux fonctions : l'une, faisant intervenir un premier domaine de liaison, permettrait l'interaction avec les cellules endothéliales et l'extravasation ; l'autre, liée à la migration au travers de la matrice extra-cellulaire, impliquerait le domaine de liaison à l'acide hyaluronique [12]. La figure 4 résume les rôles proposés pour les 


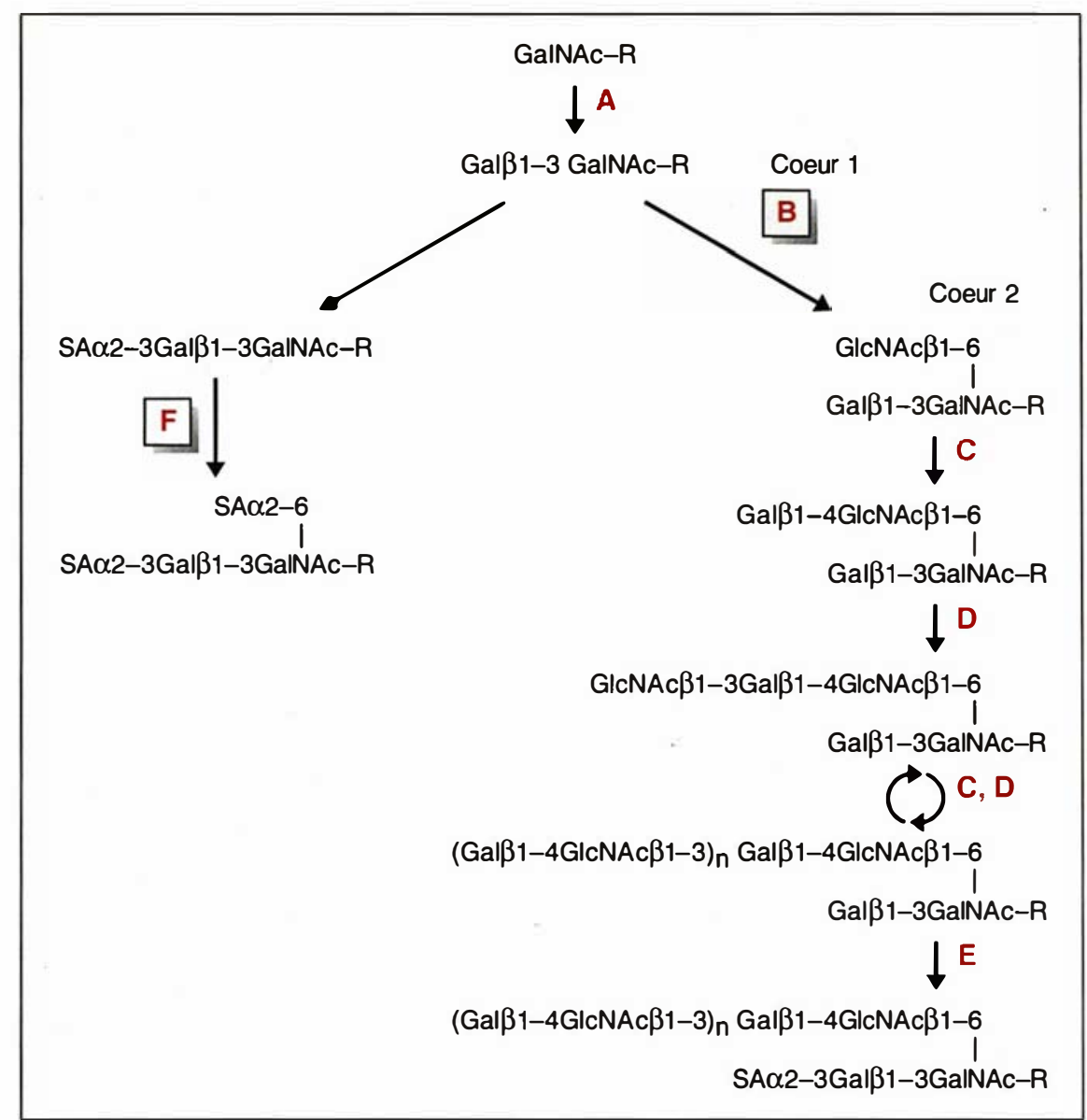

Figure 5. Voie de synthèse des oligosaccharides O-liés "cœur 1 " et " cœur 2 " lors du syndrome de Wiskott-Aldrich. $A$ : $\beta 3$ Gal-transférase; $B$ : "cœur 2 " GlcNAc transférase ; $C$ : $\beta 4$ Gal-transférase ; $D: \beta 3$ GlcNActransférase ; $E: \alpha 2-3$ sialyl-tranférase ; $F: \alpha 2-6$ sialyl-transférase II. Les activités transférasiques " $B$ " et " $F$ " encadrées sont affectées dans le syndrome de Wiskott-Aldrich; elles sont exclusives de chacune des deux voies de synthèse. L'activité "cœur 2 GIcNAc transférase" (B) des lymphocytes $T$ et des plaquettes est trois fois plus élevée chez les sujets atteints du syndrome de Wiskott-Aldrich que chez les sujets normaux. L'activité "cœur 2 GlcNAc transférase " (B) des cellules $B$ immortalisées par le virus d'EpsteinBarr est beaucoup plus faible chez les sujets atteints du syndrome de WiskottAldrich que chez les sujets normaux; I'inverse se produit pour l'a2-6 sialyltransférase II. (D'après [15.])

sélectines et H-CAM lors de la domiciliation (homing) des lymphocytes.

\section{Intégrines}

Les intégrines sont des molécules d'adhérence impliquées dans les phénomènes de reconnaissance cellule/matrice extracellulaire ou cellule/cellule. Ces glycoprotéines sont des hétérodimères dont la chaîne $\beta$ définit la famille. Les intégrines qui nous intéresseront ici sont de la famille $\beta 2$ (CD 18). important dans les phénomènes de reconnaissance cellulaire concernant le système immunitaire. Ces deux intégrines, bien que ne différant que par leur chaîne $\alpha$, reconnaissent des domaines différents d'ICAM1 : LFA1 reconnaissant le premier domaine NH2-terminal analogue aux immunoglobulines et Mac1, le troisième. Il vient d'être démontré que l'adhérence de Mac1 à ICAM1 peut être modulée par la $\mathrm{N}$-glycosylation de cette dernière. En effet, l'équipe de T. A. Springer a pu montrer que le raccourcissement ou la suppression de chaînes oligosaccharidiques N-liées d'ICAM1 augmentent l'affinité de cette molécule pour Mac1, sans modifications de celle-ci pour LFA1. Pour cela, ont été utilisés soit des inhibiteurs de l' $\alpha$-mannosidase-1, telle la déoxymannojirimycine, de façon à bloquer la maturation des structures oligosaccharidiques $\mathrm{N}$-liées au niveau des formes polymannosidiques, soit la mutagenèse dirigée pour supprimer certaines séquences signal Asn-X-Ser ou Thr et, par conséquent, des châ̂nes oligosaccharidiques. De plus, l'adhérence de polynucléaires neutrophiles exprimant conjointement LFA1 et Mac1 à une forme soluble d'ICAM1 (forme tronquée au niveau du fragment hydrophobe transmembranaire) exprimée par différents types cellulaires de façon à obtenir des glycosylations variables, a permis de démontrer que les molécules d'ICAM1 portant des structures oligosaccharidiques complexes se lient préférentiellement à LFA1, alors que celles qui ne portent pas d'oligosaccharides N-liés, ou seulement des formes polymannosidiques, préfèrent Mac1 [13].

\section{Importance en pathologie}

En dehors des phénomènes de recirculation ou homing, les structures glucidiques portées par certains antigènes leucocytaires pourraient être impliquées dans plusieurs autres phénomènes immunologiques, notamment en ce qui concerne la fonction de certaines intégrines. Les intégrines leucocytaires (leukocyte function associated 1) LFA1 (CD11a/CD18) et Mac1 (CD11b/CD18) ont pour ligand une molécule de la superfamille des immunoglobulines, ICAM1 (intercellular adhesion molecule), et jouent un rôle
Il est également connu que les molécules HLA de classe 2 expriment, chez un même individu, une glycosylation variable qui est fonction du type cellulaire (lymphocytes versus monocytes) [14]. Mais surtout, une affection héréditaire récessive liée au chromosome $\mathrm{X}$ - le syndrome de Wiskott-Aldrich, caractérisé notamment par un profond déficit immunitaire - témoigne particulièrement de l'importance de la glycosylation dans le développement de la réponse 
immunitaire. En effet, les lymphocytes et les plaquettes des sujets atteints de ce syndrome ont de profondes anomalies de la O-glycosylation, apparemment restreintes aux cellules hématopoïétiques. Ces anomalies auraient pour origine une régulation anormale de l'expression de deux activités glycosyltransférasiques, celle de la " cœur 2 GlcNactransférase " et celle de l' $\alpha 2-6$ sialyl transférase 2, la première permettant la fixation d'un résidu de $\mathrm{N}$-acétylglucosamine conduisant à la création de structures de type polylactosamines et la seconde permettant de sialyler en 2-6 une structure déjà sialylée en $\alpha 2-3$ (figure 4). Ainsi les lymphocytes $\mathrm{T}$ et les plaquettes des malades atteints du syndrome de Wiskott-Aldrich ont une activité " cœur 2 GlcNActransférase " trois fois plus élevée que celle des sujets normaux. Après stimulation des lymphocytes $\mathrm{T}$ par des anticorps anti-CD 3, on observe une inversion des phénotypes, division par trois de l'activité chez les malades et augmentation par trois chez les sujets normaux. Les travaux effectués sur les cellules B transformées par le virus d'Epstein-Barr, provenant de sujets normaux ou atteints du syndrome de Wiskott-Aldrich, mettent en évidence une activité " cœur 2 GlcNActransférase " beaucoup plus faible et une activité " sialyltransférase $\alpha$ 2-6" beaucoup plus forte chez les sujets atteints que chez les sujets normaux, alors que onze autres activités glycosyltransférasiques ne varient pas. Ces modifications d'activité ont un retentissement sur la glycosylation des glycoprotéines membranaires, qui n'a pour l'instant été recherché et trouvé que pour la leucosialine (CD43) [15], mais d'autres glycoprotéines pourraient subir des modifications de glycosylation au cours de cette affection.

Celles-ci peuvent entraîner des variations fonctionnelles, comme nous l'avons montré pour le transporteur du glucose [16]. Reste cependant à connaître la ou les protéines modifiées et à élucider par quels mécanismes elles pourraient perturber la réponse immunitaire. Il nous faudra également savoir comment une altération génétique, située sur la partie proximale du bras court du chromosome $\mathrm{X}$, altère la régulation d'activités transférasiques dans les cellules des lignées hématopoïétiques.

Enfin, il a été montré que l'anémie congénitale dysérythropoïétique de type 2 ou HEMPAS, une anémie rare d'origine génétique transmise selon un mode autosomique récessif, était due à un déficit en $\alpha$ mannosidase-2, une enzyme nécessaire à la maturation des structures glycaniques N-liées [17].

\section{MALADIES IMMUNO-HÉMATOLOGIQUES LIÉES Ä DES ANOMALIES DE LA GLYCOSYLATION}

Le syndrome de Wiskott-Aldrich est une déficience immunitaire mixte affectant l'immunité humorale et cellulaire associée à une thrombopathie. Le déficit immunitaire de type humoral associe le plus souvent une baisse des IgM et parfois des IgG à une élévation du taux des IgE et IgA sériques. Le déficit immunitaire cellulaire progressif aboutit, quant à lui, en quelques années, à une lymphopénie prédominant sur les populations de cellules $T$. Le tableau clinique associe un eczéma chronique à des infections bactériennes, mycosiques, virales et parasitaires. Le pronostic est sombre, aggravé encore par les risques hémorragiques liés à la thrombopathie, et à la fréquence anormale des leucémies et des cancers chez ces sujets.

$L$ 'anémie dysérythropoïétique de type II OU HEMPAS (hereditary erythroblastic multinuclearity with positive acidified serum lysis test) est une anémie rare à transmission autosomique récessive, caractérisée par une érythropoièse inefficace. La moelle osseuse des sujets atteints présente un grand nombre d'érythroblastes (hyperplasie érythroïde) dont certains, les plus mûres, sont polynucléés. Les hématies mûres ont une acanthocytose, c'est-à-dire des déformations à type de villosités ou de pseudopodes. L'anémie est associée à une splénomégalie, parfois à une hépatomégalie, ou à une hémosidérose, et à des malformations de la boîte crânienne.

Ces faits expliquent le regain d'intérêt pour l'étude du rôle des glycoconjugués dans la physiopathologie des cellules sanguines, plus particulièrement en ce qui concerne le système immunitaire.

Ainsi, de nouvelles voies s'ouvrent à la glycobiologie ; l'étape de la caractérisation des structures passée, l'essor des techniques de la biologie cellulaire et moléculaire devrait nous permettre d'aborder enfin la physiopathologie de ces structures omniprésentes à la surface des cellules

\section{Summary}

Glycobiology and blood cells

Glycobiology, the study of the biology of carbohydrates and glycoconjugates, has recently benefited from a renewed interest. Indeed, results of structural studies performed during the past years can now be used for investigating of the physiological roles of glycoconjugates, particulary in the field of immunobiology and hematology. Recent studies on leukocyte adhesion have led to the characterization of three lectins of the same family, the selectins or LECCAMs (L for lectin, $\mathrm{E}$ for epidermal growth factor-like, $\mathrm{C}$ for complement-like short consensus repeat, CAM for cell adhesion molecule). L-selectin (LEC-CAM 1) is involved in lymphocyte homing while E-selectin (LECCAM 2 or ELAM 1) and Pselectin (LEC-CAM 3, GMP 140 or CD 62) play an important role in leukocyte adhesion to endothelia and platelets during inflammation and blood clotting. Another adhesion molecule termed $\mathrm{H}$ CAM (CD44 or Hermes antigen) plays also a role in lymphocyte homing by binding to carbohydrate determinants on extracellular matrix. Moreover, some hematological or immunological diseases as Wiskott-Aldrich syndrom or congenital dyserythropoïetic anemia type 2 are related to glycosylation abnormalities or defects. 